Article

\title{
Whole Tumor Histogram Analysis Using DW MRI in Primary Central Nervous System Lymphoma Correlates with Tumor Biomarkers and Outcome
}

\author{
Insun Chong ${ }^{1}{ }^{\mathbb{D}}$, Quinn Ostrom ${ }^{2}$, Bilal Khan ${ }^{1}$, Dima Dandachi ${ }^{3}$, Naveen Garg ${ }^{4}$, \\ Aikaterini Kotrotsou 4,5 (D), Rivka Colen ${ }^{4,5}$ and Fanny Morón ${ }^{1, *}$ \\ 1 Department of Radiology, Baylor College of Medicine, Houston, TX 77030, USA; \\ insunchong@icloud.com (I.C.); bilal.khan@bcm.edu (B.K.) \\ 2 Section of Epidemiology and Population Sciences, Department of Medicine, Dan L Duncan Comprehensive \\ Cancer Center, Baylor College of Medicine, Houston, TX 77030, USA; quinn.ostrom@bcm.edu \\ 3 Division of Infectious Disease, Department of Medicine, University of Missouri-Columbia, \\ Columbia, MO 65212, USA; dandachid@health.missouri.edu \\ 4 Department of Diagnostic Radiology, The University of Texas MD Anderson Cancer Center, \\ Houston, TX 77030, USA; ngarg@mdanderson.org (N.G.); aikaterini.kotrotsou@gmail.com (A.K.); \\ rcolen@mdanderson.org (R.C.) \\ 5 Department of Cancer Systems Imaging, The University of Texas MD Anderson Cancer Center, \\ Houston, TX 77030, USA \\ * Correspondence: fmoron@bcm.edu
}

Received: 1 August 2019; Accepted: 2 October 2019; Published: 8 October 2019

Abstract: The ability to non-invasively predict outcomes and monitor treatment response in primary central nervous system lymphoma (PCNSL) is important as treatment regimens are constantly being trialed. The aim of this study was to assess the validity of using apparent diffusion coefficient (ADC) histogram values to predict Ki-67 expression, a tumor proliferation marker, and patient outcomes in PCNSL in both immunocompetent patients and patients living with HIV (PLWH). Qualitative PCNSL magnetic resonance imaging (MRI) characteristics from 93 patients (23 PLWH and 70 immunocompetent) were analyzed, and whole tumor segmentation was performed on the ADC maps. Quantitative histogram analyses of the segmentations were calculated. These measures were compared to PCNSL Ki-67 expression. Progression-free survival (PFS) and overall survival (OS) were analyzed via comparison to the International Primary Central Nervous System Lymphoma Collaboration Group Response Criteria. Associations between ADC measures and clinical outcomes were assessed using univariate and multivariate Cox proportional hazards models. Normalized ADC $(\mathrm{nADC})_{\text {Min }}, \mathrm{nADC}_{\text {Mean }}, \mathrm{nADC}_{1}, \mathrm{nADC}_{5}$, and $\mathrm{nADC}_{15}$ values were significantly associated with a poorer OS. $\mathrm{nADC}_{\text {Max }}, \mathrm{nADC}_{\text {Mean }}, \mathrm{nADC}_{5}, \mathrm{nADC}_{15}, \mathrm{nADC}_{75}, \mathrm{nADC}_{95}, \mathrm{nADC}_{99}$ inversely correlated with Ki-67 expression. OS was also significantly associated with lesion hemorrhage. PFS was not significantly associated with ADC values but with lesion hemorrhage. ADC histogram values and related parameters can predict the degree of tumor proliferation and patient outcomes for primary central nervous system lymphoma patients and in both immunocompetent patients and patients living with HIV.

Keywords: primary central nervous system lymphoma; magnetic resonance imaging; diffusion-weighted imaging; apparent diffusion coefficient; patients living with HIV; Ki-67

\section{Introduction}

Primary central nervous system lymphoma (PCNSL) represents a rare, aggressive subgroup of Non-Hodgkin Lymphoma [1,2]. Although the majority of the cases of PCNSL are sporadic, 
a minority are associated with immunosuppression, such as in patients living with HIV (PLWH) [1-3]. Treatment regimens for PCNSL remain restricted due to the limited number of agents that can penetrate the blood-brain barrier. Development of an ideal treatment regimen for PCNSL is complex as PCNSL in PLWH is a distinct entity from other central nervous system (CNS) neoplasms as it is associated with the Epstein-Barr virus, which allows for the additional possibility of antiviral-based treatment regimens [1-4].

$\mathrm{Ki}-67$ is a proliferation marker that has been evaluated in lymphomas and other malignancies [5-7]. Some studies have shown that the proportion of malignant cells positively stained for Ki-67 may have prognostic importance in PCNSL and correlate with clinical outcomes [8,9].

Identifying reliable methods of non-invasively predicting outcomes is an important area of research as new treatment regimens in both immunocompetent patients and PLWH are constantly being investigated [10-14]. Magnetic resonance imaging (MRI) is the modality of choice for imaging PCNSL, although definitive diagnosis requires histology [15-18]. Diffusion weighted imaging (DWI) and corresponding apparent diffusion coefficient (ADC) maps can reflect the microscopic cellular environment $[10,19]$. ADC histogram values have been reported to predict tumor cellularity in a broad range of neoplasms including lymphomas $[10,14,19]$. Furthermore, ADC values have also been shown to correspond to tumor markers such as Ki-67, predict survival, and serve as an effective means of monitoring treatment response in PCNSL [12,13,20-23]; however, these prior studies were limited by smaller sample sizes, using single regions of interests to obtain ADC values rather than using more accurate whole tumor segmentation, and the absence of data on PLWH, a patient population classically affected by PCNSL [22,24].

Therefore, our goal was to describe and compare the imaging findings of PCNSL for PLWH and immunocompetent patients, the relationship between Ki-67 expression and ADC values, and the relationship between prognosis and ADC values.

\section{Results}

\subsection{Patient Demographics}

We retrospectively studied patients with PCNSL who were $>18$-years-old and had brain parenchymal PCNSL that presented to The University of Texas MD Anderson Cancer Center (MDACC) and Ben Taub Hospital (BTH) during the study period, who had no evidence of systemic lymphoma by whole-body computed tomography or positron emission tomography scan, and bone marrow biopsy. We excluded patients with diffuse lymphoma with CNS involvement or a relapse in the CNS, patients without pathology-proven PCNSL, and patients who had no preoperative brain MRI or had suboptimal imaging. Subsequently, we identified 93 patients, of which $32(34.4 \%)$ were from BTH and $61(65.6 \%)$ were from MDACC. Forty-nine patients (52.7\%) were non-Hispanic/white, 21 (22.6\%) were African American, 20 (21.5\%) were Hispanic and 3 (3.2\%) were Asian. Twenty-three patients $(24.7 \%)$ were human immunodeficiency virus (HIV)-positive and 70 (75.3\%) were HIV-negative. Of the African American patients, $65 \%$ were HIV-positive. Further patient demographic information is summarized in Tables 1 and 2. 
Table 1. Patient information and qualitative imaging characteristics. Results are considered significant $\left(^{*}\right)$ when $p<0.05$.

\begin{tabular}{|c|c|c|c|c|c|c|c|}
\hline & All Patients (\% of All Patients) & Deaths & Median Survival in Months (95\% CI) & $p$-Value ${ }^{\mathrm{a}}$ & Relapses & Median PFS in Months $(95 \% \mathrm{CI})$ & $p$-Value ${ }^{\mathrm{b}}$ \\
\hline Total & 93 & 55 & $41(23-76)$ & & 57 & $11(6-33)$ & \\
\hline \multicolumn{8}{|l|}{ Study site } \\
\hline BTH & $32(34.4 \%)$ & 20 & $15\left(5-^{* *}\right)$ & ref & 23 & $6(3-15)$ & ref \\
\hline MDACC & $61(65.6 \%)$ & 35 & $47(37-1001)$ & $0.04 *$ & 34 & $15(8-* *)$ & $0.04 *$ \\
\hline \multicolumn{8}{|l|}{ Gender } \\
\hline Male & $51(54.8 \%)$ & 34 & $37(11-52)$ & ref & 34 & $7(4-23)$ & ref \\
\hline Female & $42(45.2 \%)$ & 21 & $100(16-* *)$ & 0.07 & 23 & $13\left(8-^{* *}\right)$ & 0.19 \\
\hline \multicolumn{8}{|l|}{ Age } \\
\hline$<60$ & $50(53.8 \%)$ & 30 & $43\left(15-^{* *}\right)$ & ref & 31 & $11(6-* *)$ & ref \\
\hline$\geq 60$ & $43(46.2 \%)$ & 25 & $43\left(111^{* *}\right)$ & 0.75 & 26 & $11(5-* *)$ & 0.82 \\
\hline \multicolumn{8}{|l|}{ Race/Ethnicity } \\
\hline Non-Hispanic/White & $49(52.7 \%)$ & 33 & $43(11-76)$ & ref & 31 & $11(6-* *)$ & ref \\
\hline African-American & $21(22.6 \%)$ & 14 & $9(5-* *)$ & 0.46 & 12 & $6(3-* *)$ & 0.90 \\
\hline Hispanics & $20(21.5 \%)$ & 7 & $* *$ & 0.25 & 12 & $15\left(10^{* *}\right)$ & 0.74 \\
\hline Asians & $3(3.2 \%)$ & 1 & $16\left(16-^{* *}\right)$ & 0.67 & 2 & $6\left(5-^{* *}\right)$ & 0.86 \\
\hline \multicolumn{8}{|l|}{ HIV status } \\
\hline Positive & $23(24.7 \%)$ & 15 & $6\left(2-^{* *}\right)$ & ref & 15 & $6\left(2-^{* *}\right)$ & ref \\
\hline Negative & $70(75.3 \%)$ & 40 & $45(37-101)$ & $0.02 *$ & 42 & $11\left(8^{* * *}\right)$ & 0.26 \\
\hline \multicolumn{8}{|l|}{ ECOG } \\
\hline $0-1$ & $54(58.1 \%)$ & 26 & $47\left(43-^{* *}\right)$ & ref & 29 & $24\left(11-^{* *}\right)$ & ref \\
\hline $2-4$ & $38(40.9 \%)$ & 28 & $7(4-37)$ & $<0.001 *$ & 28 & $5.5(2-14)$ & $0.02 *$ \\
\hline \multicolumn{8}{|l|}{ Number of lesions } \\
\hline Single & $43(46.2 \%)$ & 29 & $43(24-76)$ & ref & 29 & $8(5-33)$ & ref \\
\hline Multiple & $50(53.8 \%)$ & 26 & $41(9-* *)$ & 0.91 & 28 & $14(6-* *)$ & 0.41 \\
\hline \multicolumn{8}{|l|}{ Location } \\
\hline Deep brain & $48(51.6 \%)$ & 25 & $41(23-* *)$ & ref & 29 & $11(6-* *)$ & ref \\
\hline Not deep brain & $45(48.4 \%)$ & 30 & $43(11-101)$ & 0.74 & 28 & $11(5-* *)$ & 0.93 \\
\hline \multicolumn{8}{|l|}{ Hemorrhage } \\
\hline Yes & $46(49.5 \%)$ & 32 & $15(6-52)$ & ref & 33 & $5.5(4-15)$ & ref \\
\hline No & $47(50.5 \%)$ & 23 & $76\left(41^{-* *}\right)$ & $0.004 *$ & 24 & $17\left(11^{* *}\right)$ & $0.03 *$ \\
\hline \multicolumn{8}{|l|}{ Enhancement } \\
\hline None & $1(1.1 \%)$ & - & - & - & - & - & - \\
\hline Ring & $16(17.2 \%)$ & 11 & $5\left(2-^{* *}\right)$ & ref & 10 & $5\left(2-^{* *}\right)$ & ref \\
\hline Solid & $76(81.7 \%)$ & 44 & $43(27-100)$ & 0.11 & 47 & $11(7-36)$ & 0.53 \\
\hline
\end{tabular}


Table 1. Cont

\begin{tabular}{|c|c|c|c|c|c|c|c|}
\hline & All Patients (\% of All Patients) & Deaths & Median Survival in Months (95\% CI) & $p$-Value ${ }^{\mathrm{a}}$ & Relapses & Median PFS in Months (95\% CI) & $p$-Value $^{\mathrm{b}}$ \\
\hline \multicolumn{8}{|l|}{ Type of surgery } \\
\hline Biopsy & $60(65.9 \%)$ & 35 & $43(37-101)$ & ref & 35 & $13(6-* *)$ & ref \\
\hline Partial resection & $22(24.2 \%)$ & 13 & $15(9-* *)$ & 0.689 & 15 & $9.5\left(6-^{* *}\right)$ & 0.610 \\
\hline Complete resection & $8(8.8 \%)$ & 4 & $24(3-* *)$ & 0.863 & 4 & $11(2-* *)$ & 0.912 \\
\hline Other & $1(1.1 \%)$ & 1 & $16(* *-* *)$ & 0.446 & 1 & $5(* *-* *)$ & 0.359 \\
\hline \multicolumn{8}{|l|}{ Initial Treatment } \\
\hline Supportive/Palliative & $5(5.4 \%)$ & 5 & $1\left(1-^{* *}\right)$ & ref & 5 & $1\left(1-^{* *}\right)$ & ref \\
\hline Whole brain radiation (WBRT) & $18(19.4 \%)$ & 11 & $24(3-* *)$ & $<0.001 *$ & 10 & $6(3-* *)$ & $<0.001$ * \\
\hline MTX monotherapy & $6(6.5 \%)$ & 6 & $6\left(3^{-* *}\right)$ & $0.008 *$ & 6 & $2.5(2-* *)$ & $0.048 *$ \\
\hline MTX-based combination chemo & $51(54.8 \%)$ & 28 & $47\left(35^{-* *}\right)$ & $<0.001 *$ & 31 & $13(9-* *)$ & $<0.001 *$ \\
\hline WBRT and MTX based chemo & $13(14.0 \%)$ & 5 & $100(47-* *)$ & $<0.001 *$ & 5 & $* *$ & $<0.001$ * \\
\hline \multicolumn{8}{|l|}{ Stem Cell Transplant (SCT) } \\
\hline Auto-HSCT & $9(9.7 \%)$ & 3 & $76(47-* *)$ & ref & 3 & ** & ref \\
\hline No SCT & $84(90.3 \%)$ & 52 & $37(11-53)$ & 0.10 & 54 & $8(5-15)$ & 0.06 \\
\hline
\end{tabular}


Table 2. Patient information and qualitative imaging characteristics comparing HIV-positive with HIV-negative patients. Results considered significant $\left(^{*}\right)$ when $p<0.05$.

\begin{tabular}{|c|c|c|c|}
\hline & $\begin{array}{c}\text { HIV Positive } \\
\text { (\% of All HIV+ Patients) }\end{array}$ & $\begin{array}{c}\text { HIV Negative } \\
\text { (\% of All HIV- Patients) }\end{array}$ & $p$-Value ${ }^{\text {a }}$ \\
\hline Total & $23(25 \%)$ & $70(70 \%)$ & - \\
\hline Study site & & & $<0.001 *$ \\
\hline BTH & $22(95.7 \%)$ & $10(14.3 \%)$ & \\
\hline MDACC & $1(4.3 \%)$ & $60(85.7 \%)$ & \\
\hline Gender & & & 0.16 \\
\hline Male & $16(69.6 \%)$ & $35(50.0 \%)$ & \\
\hline Female & $7(30.4 \%)$ & $35(50.0 \%)$ & \\
\hline Age & & & $<0.001 *$ \\
\hline$<60$ & $23(100.0 \%)$ & $27(38.6 \%)$ & \\
\hline$\geq 60$ & $0(0.0 \%)$ & $43(61.4 \%)$ & \\
\hline Race/Ethnicity & & & $<0.001 *$ \\
\hline Non-Hispanic/White & $2(8.7 \%)$ & $47(67.1 \%)$ & \\
\hline African-American & $15(65.2 \%)$ & $6(8.6 \%)$ & \\
\hline Hispanics & $6(26.1 \%)$ & $14(20.0 \%)$ & \\
\hline Asians & $0(0.0 \%)$ & $3(4.3 \%)$ & \\
\hline \multicolumn{4}{|l|}{ HIV status } \\
\hline Positive & - & - & \\
\hline Negative & - & - & \\
\hline ECOG & & & $<0.001 *$ \\
\hline $0-1$ & $4(17.4 \%)$ & $50(71.4 \%)$ & \\
\hline $2-4$ & $19(82.6 \%)$ & $19(27.1 \%)$ & \\
\hline Number of lesions & & & $0.046^{*}$ \\
\hline Single & $6(26.1 \%)$ & $37(52.9 \%)$ & \\
\hline Multiple & $17(73.9 \%)$ & $33(47.1 \%)$ & \\
\hline Location & & & 0.86 \\
\hline Deep brain & $11(47.8 \%)$ & $37(52.9 \%)$ & \\
\hline Not deep brain & $12(52.2 \%)$ & $33(47.1 \%)$ & \\
\hline Hemorrhage & & & $0.047^{*}$ \\
\hline Yes & $16(69.6 \%)$ & $30(42.9 \%)$ & \\
\hline No & $7(30.4 \%)$ & $40(57.1 \%)$ & \\
\hline Enhancement & & & $<0.001 *$ \\
\hline None & $1(4.3 \%)$ & $0(0.0 \%)$ & \\
\hline Ring & $13(56.5 \%)$ & $3(4.3 \%)$ & \\
\hline Solid & $9(39.1 \%)$ & $67(95.7 \%)$ & \\
\hline Type of surgery & & & 1.00 \\
\hline Biopsy & $15(68.2 \%)$ & $45(65.2 \%)$ & \\
\hline Partial resection & $5(22.7 \%)$ & $17(22.7 \%)$ & \\
\hline Complete resection & $2(9.1 \%)$ & $6(8.7 \%)$ & \\
\hline Other & $0(0.0 \%)$ & $1(1.4 \%)$ & \\
\hline Initial Treatment & & & $<0.001 *$ \\
\hline Supportive/Palliative & $3(13.0 \%)$ & $2(2.9 \%)$ & \\
\hline Whole brain radiation (WBRT) & $16(69.6 \%)$ & $2(2.9 \%)$ & \\
\hline MTX monotherapy & $0(0.0 \%)$ & $6(8.6 \%)$ & \\
\hline MTX-based combination chemo & $4(17.4 \%)$ & $47(67.1 \%)$ & \\
\hline WBRT and MTX based chemo & $0(0.0 \%)$ & $13(18.6 \%)$ & \\
\hline Stem Cell Transplant (SCT) & & & 0.11 \\
\hline Auto-HSCT & $0(0.0 \%)$ & $9(12.9 \%)$ & \\
\hline No SCT & $23(100.0 \%)$ & $61(87.1 \%)$ & \\
\hline
\end{tabular}

a $p$-value denotes statistically significant difference in characteristics between $\mathrm{HIV}-$ and $\mathrm{HIV}+$ patients. BTH = Ben Taub Hospital; MDACC = MD Anderson Cancer Center; HIV = human immunodeficiency virus; ECOG = Eastern Cooperative Oncology Group; MTX = methotrexate; HSCT = Hematopoietic stem cell transplantation. 


\subsection{Qualitative Imaging Characteristics}

Qualitative imaging characteristics were reviewed by one of the authors (F.E.M.) who is a board-certified neuroradiologist. The presence of ring enhancement was higher for PLWH (56.5\%) compared to the HIV-negative subset $(4.3 \%$ ), which was statistically significant (Table 2, $p<0.001$ ). Additionally, the presence of multiple lesions was found to be higher in the PLWH population (73.9\%) compared to the HIV-negative subset $(47.1 \%)$, which was also statistically significant $(p=0.046)$. Lesion macrohemorrhage, identified as areas of gradient-recalled echo (GRE) or susceptibility-weighted imaging (SWI) hypointensity and/or T1 shortening on the non-contrast T1-weighted imaging, was associated with HIV status $(p=0.047)$ as PLWH had a lesion hemorrhage rate of $69.6 \%$ versus $42.9 \%$ in patients with a HIV-negative status.

\subsection{Correlations between ADC Values and Ki-67 Expression}

Ki-67 expression of lesions were determined via histopathological analyses of samples obtained by biopsy, partial resection, or complete resection (percentages provided in Table 2). Apparent diffusion coefficient (ADC) values were obtained by performing whole tumor segmentation (Figure 1) using 3D Slicer (version 4.7, SlicerSolutions, Boston, MA, United States), which was done by one of the authors (I.C.) [25]. If a patient had multiple lesions, the largest lesion was used for segmentation and analysis. If applicable, lesion hemorrhage was also excluded prior to segmentation. These segmentation volumes of interest (VOIs) were reviewed by two board-certified neuroradiologists (F.E.M. and R.R.C.) in consensus (i.e., simultaneously). Whole tumor segmentation was performed to allow for the evaluation of the entire hypercellular volume of interest's intra-tumor heterogeneity, rather than a single slice region of interest placement (summary of the mean values of tumor ADC histogram parameters provided in Table S1) [22]. Among the subset of patients with available Ki-67 expression data ( 28 of 93), the relationships between Ki-67 expression and ADC parameters were explored. As compared to the larger dataset, these patients were largely from MDACC (92.9\%), HIV- (92.9\%), older (60.7\% over 60 years old), and received biopsy ( $78.6 \%$, as compared to $17.9 \%$ with partial resection and $3.6 \%$ with complete resection). Various ADC parameters extracted from the segmented lesions were found to have a statistically significant correlation with Ki-67 expression (Table 3). In all patients $(n=28)$, no significant correlations were identified. However, when only lesions without hemorrhage were included $(n=18)$, nine absolute ADC parameters were identified to have positive significant correlation to Ki-67 expression, which are as follows: $\operatorname{ADC}_{\text {Min }}(p=0.01), \operatorname{ADC}_{\text {Mean }}(p=0.02)$, $\operatorname{ADC}_{1}(p=0.01), \operatorname{ADC}_{5}(p=0.01), \operatorname{ADC}_{15}(p=0.02), \operatorname{ADC}_{16}(p=0.02), \operatorname{ADC}_{75}(p=0.03), \operatorname{ADC}_{95}(p=0.04)$, and $\mathrm{ADC}_{99}(p=0.045)$ (Table S2). When values were normalized using white matter values (nADC), there were no significant associations between $\mathrm{ADC}$ values and Ki-67 expression, although the values correlated inversely (Table 3).

Table 3. Correlation with Ki-67 in patients with available data. Results are considered significant ${ }^{*}$ ) when $p<0.05$.

\begin{tabular}{|c|c|c|c|c|c|c|c|c|}
\hline \multirow{2}{*}{ Parameter } & \multicolumn{2}{|c|}{ All Patients $(\mathrm{n}=28)$} & \multicolumn{2}{|c|}{ No Hemorrhage $(n=18)$} & \multicolumn{2}{|c|}{$\mathrm{HIV}+(\mathrm{n}=2)$} & \multicolumn{2}{|c|}{ HIV- $(n=26)$} \\
\hline & $\mathbf{r}$ & $p$-Value & $\mathbf{r}$ & $p$-Value & $\mathbf{r}$ & $p$-Value & $\mathbf{r}$ & $p$-Value \\
\hline Skewness & 0.151 & 0.45 & 0.050 & 0.84 & - & - & 0.128 & 0.53 \\
\hline Kurtosis & 0.171 & 0.38 & 0.067 & 0.79 & - & - & 0.206 & 0.31 \\
\hline $\mathrm{nADC}_{\mathrm{Min}}$ & 0.026 & 0.90 & 0.149 & 0.56 & - & - & -0.001 & 0.99 \\
\hline $\mathrm{nADC}_{\mathrm{Max}}$ & -0.190 & 0.33 & -0.255 & 0.31 & - & - & -0.239 & 0.24 \\
\hline $\mathrm{nADC}_{\text {Mean }}$ & -0.148 & 0.45 & -0.058 & 0.82 & - & - & -0.210 & 0.30 \\
\hline $\mathrm{nADC}_{1}$ & 0.032 & 0.87 & 0.155 & 0.54 & - & - & -0.002 & 0.99 \\
\hline $\mathrm{nADC}_{5}$ & -0.023 & 0.91 & 0.118 & 0.64 & - & - & -0.052 & 0.80 \\
\hline $\mathrm{nADC}_{15}$ & -0.105 & 0.60 & 0.027 & 0.92 & - & - & -0.139 & 0.50 \\
\hline $\mathrm{nADC}_{75}$ & -0.167 & 0.40 & -0.097 & 0.70 & - & - & -0.242 & 0.23 \\
\hline $\mathrm{nADC}_{95}$ & -0.232 & 0.24 & -0.268 & 0.28 & - & - & -0.339 & 0.09 \\
\hline $\mathrm{nADC}_{99}$ & -0.195 & 0.32 & -0.232 & 0.35 & - & - & -0.290 & 0.15 \\
\hline
\end{tabular}

$\mathrm{nADC}=$ normalized apparent diffusion coefficient values. 

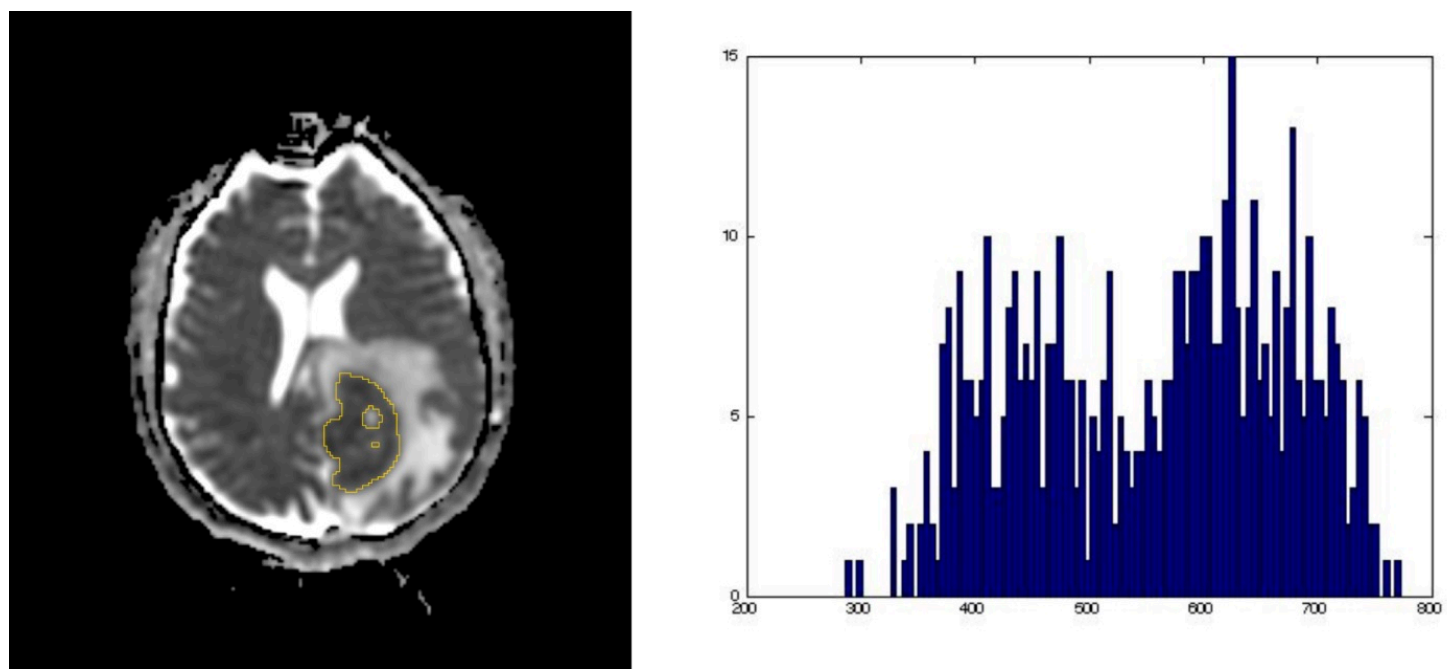

Figure 1. Representative images of an apparent diffusion coefficient (ADC) map whole tumor segmentation with its corresponding ADC histogram distribution used for data analysis.

\subsection{ADC Values and Overall Survival}

ADC values were also found to have relationships with poorer overall survival (OS) when adjusted for age, HIV status, Eastern Cooperative Oncology Group (ECOG) and treatment pattern (Table 4). Hazard ratios for OS in all patients revealed statistical significance for poorer OS with five ADC parameters generated by dividing the tumor value against the corresponding normal white matter value to correct for variations in imaging technologies within the dataset (nADC), which are as follows: $\mathrm{nADC}_{\text {Min }}(p=0.02$, Hazard Ratio $(\mathrm{HR})=0.532,95 \% \mathrm{CI}=0.294-0.963), \mathrm{nADC}_{\text {Mean }}$ $(p=0.048, \mathrm{HR}=0.689,95 \% \mathrm{CI}=0.395-1.199), \mathrm{nADC}_{1}(p=0.006, \mathrm{HR}=0.500,95 \% \mathrm{CI}=0.275-0.907)$, and $\mathrm{nADC}_{5}(p=0.02, \mathrm{HR}=0.559,95 \% \mathrm{CI}=0.314-0.996)$, and $\mathrm{nADC}_{15}(p=0.03, \mathrm{HR}=0.717$, $95 \% \mathrm{CI}=0.409-1.257)$. ECOG status and treatment pattern had a statistically significant effect on $\mathrm{OS}$ in all models. When lesions with hemorrhage were excluded, the $\mathrm{ADC}_{\mathrm{Min}}, \mathrm{nADC}_{\mathrm{Min}}, \mathrm{nADC}_{1}$, $\mathrm{nADC}_{5}, \mathrm{nADC}_{5}$ parameters were significantly associated with poorer survival, while ECOG and treatment pattern had independent statistically significant effects. When analysis was stratified by HIV status, no ADC values were associated with OS in HIV+ patients, while higher values of two $\left(\mathrm{nADC}_{\mathrm{Min}}, \mathrm{nADC}_{\text {Mean }}\right)$ were associated with poorer survival and five $\left(\mathrm{ADC}_{1}, \mathrm{nADC}_{5} \mathrm{nADC}_{15} \mathrm{nADC}_{75}\right.$ $\mathrm{nADC}_{95}$ ) were associated with improved survival in HIV- patients. ECOG status exerted a statistically significant effect on survival in HIV- patients only, but treatment significantly affected survival in both groups.

\subsection{ADC Values and Progression-Free Survival}

There was a marginally significant association between $\mathrm{nADC}_{\text {min }}$ and progression free survival (PFS) $(p=0.05, \mathrm{HR}=0.602,95 \% \mathrm{CI}=0.344-1.053)$. This association was statistically significant in HIV- patients $(p=0.03, \mathrm{HR}=0.557,95 \% \mathrm{CI}=0.295-1.052)$ and marginally significant in HIV+ patients $(p=0.06, \mathrm{HR}=0.328,95 \% \mathrm{CI}=0.067-1.603)$, and treatment pattern was statistically significant in both models (Table 5). 
Table 4. Hazard ratios for overall survival in all patients and patients without lesion hemorrhage. Results are considered significant $\left(^{*}\right)$ when $p<0.05$.

\begin{tabular}{|c|c|c|c|c|c|c|c|c|}
\hline \multirow{2}{*}{ Feature } & \multicolumn{2}{|c|}{ All Patients $(n=93)$} & \multicolumn{2}{|c|}{ No Hemorrhage $(n=47)$} & \multicolumn{2}{|c|}{ HIV+ $(n=23)$} & \multicolumn{2}{|c|}{ HIV $-(n=70)$} \\
\hline & $p$-Value ${ }^{\text {a }}$ & $\mathrm{HR}^{\mathrm{b}}(95 \% \mathrm{CI})$ & $p$-Value ${ }^{a}$ & $\mathrm{HR}^{\mathrm{b}}(95 \% \mathrm{CI})$ & $p$-Value ${ }^{a}$ & $\mathrm{HR}^{\mathrm{b}}(95 \% \mathrm{CI})$ & $p$-Value ${ }^{a}$ & $\mathrm{HR}^{\mathrm{b}}(95 \% \mathrm{CI})$ \\
\hline Skewness & 0.28 & $0.861(0.468-1.585)$ & 0.50 & $1.383(0.485-3.946)$ & 0.66 & $1.224(0.288-5.203)$ & 0.26 & $0.796(0.406-1.561$ \\
\hline Kurtosis & 0.38 & $1.443(0.745-2.794)$ & 0.82 & $1.703(0.589-4.924)$ & $0.03 *$ & $2.742(0.447-16.813)$ & 0.56 & $0.848(0.43-1.671)$ \\
\hline $\mathrm{nADC}_{\mathrm{Min}}$ & $0.02^{*}$ & $0.532(0.294-0.963)$ & $0.02 *$ & $0.425(0.143-1.265)$ & 0.10 & $0.416(0.09-1.925)$ & 0.03 * & $1.376(0.704-2.688$ \\
\hline $\mathrm{nADC}_{\mathrm{Max}}$ & 0.32 & $0.943(0.54-1.649)$ & 0.29 & $0.68(0.25-1.848)$ & 0.63 & $1.561(0.474-5.136)$ & 0.12 & 0.935 (0.469-1.864 \\
\hline $\mathrm{nADC}_{\text {Mean }}$ & $0.048^{*}$ & $0.689(0.395-1.199)$ & 0.07 & $0.3(0.097-0.93)$ & 0.61 & $1.067(0.311-3.653)$ & 0.03 * & $1.384(0.682-2.81)$ \\
\hline $\mathrm{nADC}_{1}$ & $0.006^{*}$ & $0.5(0.275-0.907)$ & $0.01 *$ & $0.286(0.087-0.945)$ & 0.18 & $0.416(0.09-1.925)$ & $0.01 *$ & $0.539(0.274-1.061$ \\
\hline $\mathrm{nADC}_{5}$ & $0.02 *$ & $0.559(0.314-0.996)$ & $0.02 *$ & $0.307(0.102-0.928)$ & 0.44 & $0.578(0.15-2.222)$ & $0.02 *$ & $0.674(0.318-1.428$ \\
\hline $\mathrm{nADC}_{15}$ & $0.03 *$ & $0.717(0.409-1.257)$ & 0.04 * & $0.377(0.12-1.184)$ & 0.55 & $1.276(0.394-4.13)$ & $0.03 *$ & $0.543(0.271-1.086$ \\
\hline $\mathrm{nADC}_{75}$ & 0.08 & $0.599(0.342-1.05)$ & 0.09 & $0.318(0.102-0.994)$ & 0.69 & $0.721(0.205-2.539)$ & 0.03 * & $0.507(0.257-1.002$ \\
\hline $\mathrm{nADC}_{95}$ & 0.12 & $0.735(0.424-1.272)$ & 0.13 & $0.396(0.125-1.255)$ & 0.97 & $0.984(0.287-3.369)$ & 0.04 * & $0.553(0.277-1.104$ \\
\hline $\mathrm{nADC}_{99}$ & 0.26 & $0.801(0.46-1.393)$ & 0.25 & $0.478(0.162-1.41)$ & 0.66 & $1.476(0.428-5.096)$ & 0.06 & $0.552(0.275-1.11)$ \\
\hline
\end{tabular}

Models adjusted for age at diagnosis, ECOG, HIV status (unless stratified by HIV status), and treatment pattern. ${ }^{\mathrm{a}} p$-value is for continuous increase of value for all ADC values. ${ }^{\mathrm{b}}$ Hazard ratio is calculated by comparing below median to above median values for all $\mathrm{ADC}$ values. $\mathrm{HR}=$ Hazard Ratio. $\mathrm{CI}=\mathrm{Confidence} \mathrm{Interval}$. $\mathrm{nADC}=$ normalized apparent diffusion coefficient values.

Table 5. Hazard ratios for progression-free survival in all patients and patients without lesion hemorrhage. Results are considered significant $\left({ }^{*}\right)$ when $\mathrm{p}<0.05$.

\begin{tabular}{|c|c|c|c|c|c|c|c|c|}
\hline \multirow{2}{*}{ Feature } & \multicolumn{2}{|c|}{ All Patients $(n=93)$} & \multicolumn{2}{|c|}{ No Hemorrhage $(n=47)$} & \multicolumn{2}{|c|}{ HIV+ $+(n=23)$} & \multicolumn{2}{|c|}{ HIV- $(n=70)$} \\
\hline & $p$-Value ${ }^{a}$ & $\mathrm{HR}^{\mathrm{b}}(95 \% \mathrm{CI})$ & $p$-Value ${ }^{a}$ & $\mathrm{HR}^{\mathrm{b}}(95 \% \mathrm{CI})$ & $p$-Value ${ }^{a}$ & $\mathrm{HR}^{\mathrm{b}}(95 \% \mathrm{CI})$ & $p$-Value ${ }^{a}$ & $\mathrm{HR}^{\mathrm{b}}(95 \% \mathrm{CI})$ \\
\hline Skewness & 0.28 & $0.77(0.43-1.379)$ & 0.22 & $0.8(0.31-2.067)$ & 0.53 & 1.355 (0.301-6.097) & 0.13 & $0.645(0.337-1.234)$ \\
\hline Kurtosis & 0.14 & $1.071(0.57-2.012)$ & 0.63 & $0.565(0.227-1.406)$ & $0.03 *$ & $2.585(0.428-15.628)$ & 0.34 & $1.015(0.522-1.974)$ \\
\hline $\mathrm{nADC}_{\mathrm{Min}}$ & 0.05 & $0.602(0.344-1.053)$ & 0.18 & $0.479(0.187-1.225)$ & 0.06 & $0.328(0.067-1.603)$ & $0.03 *$ & $0.557(0.295-1.052)$ \\
\hline $\mathrm{nADC}_{\mathrm{Max}}$ & 0.93 & $1.273(0.744-2.178)$ & 0.54 & $1.258(0.5-3.165)$ & 0.71 & $1.311(0.385-4.458)$ & 0.44 & $1.016(0.512-2.016)$ \\
\hline$n A D C_{\text {Mean }}$ & 0.45 & $0.993(0.588-1.677)$ & 0.99 & $0.952(0.382-2.373)$ & 0.42 & $0.885(0.246-3.179)$ & 0.27 & $0.839(0.45-1.566)$ \\
\hline $\mathrm{nADC}_{1}$ & 0.13 & $0.634(0.363-1.105)$ & 0.30 & $0.526(0.21-1.318)$ & 0.10 & $0.328(0.067-1.603)$ & 0.14 & $0.593(0.316-1.115)$ \\
\hline $\mathrm{nADC}_{5}$ & 0.30 & $0.761(0.445-1.3)$ & 0.48 & $0.707(0.298-1.679)$ & 0.26 & 0.507 (0.123-2.097) & 0.25 & $0.669(0.357-1.251)$ \\
\hline $\mathrm{nADC}_{15}$ & 0.41 & $0.912(0.536-1.552)$ & 0.60 & $0.793(0.315-1.993)$ & 0.33 & $1.158(0.341-3.931)$ & 0.30 & $0.697(0.369-1.318)$ \\
\hline $\mathrm{nADC}_{75}$ & 0.47 & $0.786(0.464-1.332)$ & 0.82 & $0.887(0.348-2.263)$ & 0.54 & $0.581(0.154-2.186)$ & 0.23 & $0.652(0.346-1.228)$ \\
\hline $\mathrm{nADC}_{95}$ & 0.59 & $0.919(0.543-1.555)$ & 0.58 & $1.163(0.436-3.106)$ & 0.89 & $0.82(0.229-2.941)$ & 0.24 & $0.745(0.394-1.408)$ \\
\hline $\mathrm{nADC}_{99}$ & 0.93 & $1.127(0.663-1.916)$ & 0.38 & $1.313(0.505-3.412)$ & 0.79 & $1.261(0.353-4.499)$ & 0.38 & $0.889(0.467-1.694)$ \\
\hline
\end{tabular}

Models adjusted for age at diagnosis, ECOG, HIV status (unless stratified by HIV status), and treatment pattern. a The $p$-value is for continuous increase of value for all ADC values $b$ Hazard ratio is calculated by comparing below median to above median values for all ADC values. HR = Hazard Ratio. CI $=$ Confidence Interval. $\mathrm{nADC}=$ normalized apparent diffusion coefficient values. 


\subsection{Additional Factors Impacting Overall Survival}

Along with ADC values, additional factors, including lesion hemorrhage, HIV status, treatment with auto-stem cell transplantation, and ECOG scores impacted OS (Tables 1 and 4). The absence of lesion hemorrhage was associated with better OS $(p=0.004)$. HIV status was associated with poorer OS, as the median survival time for PLWH was 6 months versus 40 months for HIV-negative patients $(p=0.02)$. Treatment with auto-stem cell transplantation was marginally associated with increased median OS with 76 months, compared to 37 months in patients who were not treated with stem cell transplantation. Lastly, ECOG scores between 2 and 4 had a median OS of 7 months versus 47 months for ECOG scores less than $2(p<0.001)$. Number of tumors, size of the largest tumor, tumor enhancement pattern, cortical invasion or deep brain involvement were not found to be associated with poorer survival outcomes.

\subsection{Additional Factors Impacting Progression-Free Survival}

Additional factors, including lesion hemorrhage $(p=0.03)$ and ECOG scores $(p=0.02)$, impacted PFS (Tables 1 and 5). Additionally, ECOG scores between 2 and 4 had median PFS of 5.5 months, which was significantly less than 24 months for ECOG scores less than $2(p=0.02)$. Periventricular location and ependymal involvement also impacted PFS in immunocompetent patients $(p=0.04, p=0.03)$. Size of the largest tumor, number of lesions, tumor enhancement pattern or HIV status were not associated with poorer PFS.

\section{Discussion}

The ability to non-invasively predict outcomes and monitor treatment response in primary central nervous system lymphoma (PCNSL) is important as new treatment regimens are constantly being trialed. We aimed to investigate how ADC histogram profiling with whole tumor segmentation reflects tumor proliferation and overall patient outcomes in both patients living with HIV and immunocompetent patients with PCNSL. Additionally, we aimed to investigate qualitative imaging characteristics of PCNSL to explore additional factors that may influence prognosis. This information may eventually allow both clinicians and radiologists to accurately analyze tumor proliferation and predict patient prognosis without the need for invasive procedures such as biopsies.

Prior studies have demonstrated significant correlations between ADC parameters and Ki-67 [6,7,12,13,20-23,26]. Our results with the normalized ADC histogram values demonstrate these same relationships but were not statistically significant. This may be due, in part, to tumor hemorrhage, and though macrohemorrhage was excluded from our lesion segmentation, microhemorrhage could not be accounted for. Tumors with hemorrhage (46 of 93 patients; 16 of $23 \mathrm{PLWH}$ ) have blood products that age heterogeneously based on contributions from the regional oxygen tension, the concentration of tissue macrophages, and the presence of tumor cells [27]. These factors result in differing levels of contributions to ADC signal from T2-relaxation, T1-relaxation, and T2*-effects, thereby resulting in an alteration of the relationship between regional cellularity and ADC [28]. Another consideration is that hemorrhage results in local cellular heterogeneity, which can cause changes in the correlations between ADC and Ki-67 [21,29]. Additionally, tumor necrosis may not have been optimally excluded as the ADC maps were not co-registered with $\mathrm{T} 1$ post-contrast images, which may be a future area for further research.

Our findings agree with prior studies that demonstrated significant associations between several ADC parameters and poorer OS when lesions with hemorrhage were excluded but also found an association between $\mathrm{nADC}_{15}$ and poorer OS [12,20-23]. Our results also agree with prior studies examining qualitative PCNSL imaging characteristics in PLWH that found a higher rate of ring enhancing lesions and lesion hemorrhage in PLWH [18,30,31]. The lack of significant associations between PCNSL ADC parameters and survival in PLWH may be due to two factors: 1) distortion of the ADC profile of the tumor due to intratumor hemorrhage resulting in alterations of the tumor 
microenvironment as previously stated and 2) inter-/intra-tumor variability with PCNSL in PLWH, due in part to lesion necrosis $[16,18,27-29,32]$. However, lesion hemorrhage was significantly associated with OS and PFS in both immunocompetent patients and PLWH [33]. Ependymal involvement and periventricular location, found to be characteristic of PCNSL, were also significantly associated with PFS in immunocompetent patients [30,31]. Relationships between hemorrhage, ependymal involvement, and periventricular location to outcomes have not been well-established in the PCNSL, indicating possible separate prognostic factors to consider in patients with PCNSL and additional control measures to be used for further research purposes [33].

However, unlike prior studies on PCNSL histogram analysis, our study further substantiates the expanding literature on the relationship between ADC values, patient outcomes, and tumor pathologic findings by (1) utilizing a larger patient population, (2) performing whole tumor segmentation rather than using single region of interest for ADC analysis, (3) performing exclusion analysis for lesion hemorrhage and necrosis, and (4) including PLWH, an important patient population affected by PCNSL. Our hope with this work is to motivate additional studies with perhaps even larger cohorts and increase validations of these relationships in order to automate the analyses of tumor proliferation and patient outcomes via integration with either a picture archiving and communication system (PACS) or a radiology informatics system and to facilitate the creation of predictive models which can be incorporated into clinical support systems.

\section{Limitations}

There are several limitations with this study. Firstly, patients were selected from only two institutions, both in the same city. Secondly, the patients were scanned using several different machines, although this confounding effect was minimized by using ADC values of normal white matter from the side contralateral to the tumor, a method that has been previously reported $[10,12,23]$. Thirdly, the degree of patient exclusion was a limitation of this study. While the sample size of the study was relatively larger than prior studies, lesions with hemorrhage were further excluded for portions of the analysis. This presents a potential limitation in using ADC histogram analysis for patients with PCNSL complicated by lesion hemorrhage. Lastly, the biopsied portion of PCNSL specimen used to calculate Ki-67 expression may not be representative of its expression throughout the whole tumor and may decrease the accuracy of the statistical analysis.

\section{Materials and Methods}

\subsection{Patient Selection and Review}

We conducted a Baylor College of Medicine IRB-approved (H-39346, 27 February 2018), Health Insurance Portability and Accountability Act compliant (HIPAA) retrospective study of patients diagnosed with PCNSL at the University of Texas MD Anderson Cancer Center between March 2000 and July 2016 and at Ben Taub Hospital, between January 2012 and December 2016. All the patients that met the following criteria were included: (1) age $>18$ years; (2) patients with brain parenchymal PCNSL that presented to MDACC and BTH during the study period, who had no evidence of systemic lymphoma by whole-body computed tomography or positron emission tomography scan and bone marrow biopsy. Exclusion criteria were (1) patients with diffuse lymphoma with CNS involvement or relapse in the CNS, (2) patients without pathology proven PCNSL, and (3) patients who had no preoperative brain MRI or had suboptimal preoperative imaging. We reviewed the medical records and collected information on socio-demographic characteristics (e.g., gender, race, ethnicity), HIV status, biopsy results, immunohistochemical staining (including Ki-67), and clinical outcomes. Due to the retrospective nature of this analysis, not all measures were available on all individuals. 


\subsection{MR Imaging Protocols}

All images were acquired within the clinical diagnostic parameters using either a 1.5 or 3.0 Tesla GE (Milwaukee, WI, USA) or Siemens (Erlangen, Germany) scanner with the corresponding head coils. Diffusion-weighted imaging (DWI) images were obtained with the following parameters using spin echo and spin echo planar sequences: (1) for BTH: minimum Echo time (TE), 8000 ms repetition time (TR), no flip angle, $5 \mathrm{~mm}$ slice thickness (27 slices), and $240 \mathrm{~mm}^{2}$ field of view (FOV) with diffusion-sensitizing gradients applied with $b$ factors of 0 and $1000 \mathrm{~s} / \mathrm{mm}^{2}$ and (2) for MDACC: $8.9 \mathrm{~ms}$ $\mathrm{TE}, 6600 \mathrm{~ms}$ TR, $5 \mathrm{~mm}$ slice thickness, and $230 \mathrm{~mm}^{2} \mathrm{FOV}$ with diffusion-sensitizing gradients applied with $\mathrm{b}$ factors of 0,500 , and $1000 \mathrm{~s} / \mathrm{mm}^{2}$. ADC maps were automatically generated by the operating console of the magnetic resonance (MR) scanner.

\subsection{Analysis of MRI Imaging}

For image analysis and segmentation, we used 3D Slicer (version 4.7, SlicerSolutions, Boston, MA, United States), an open source software platform for medical imaging informatics [25]. Images were exported from the picture archiving and communication system (PACS) in Digital Imaging and Communications in Medicine (DICOM) format and converted to a Neuroimaging Informatics Technology Initiative (NIfTI) file. The volume of interest (VOI) consisting of areas of ADC-restriction in a biopsy-proven tumor were outlined by one of the authors (I.C.) (Figure 1). If a patient had multiple lesions, the largest lesion was segmented and used for analysis. Regions of macrohemorrhage, identified as areas of gradient-recalled echo (GRE) or susceptibility-weighted imaging (SWI) hypointensity and/or $\mathrm{T} 1$ shortening on the non-contrast T1-weighted imaging, were excluded from the segmentation volumes. A normal white matter region of interest in the contralateral hemisphere was obtained for normalization purposes in order to account for any scanner variability [10,34]. The segmented images were reviewed in consensus (i.e., simultaneously) by two board-certified neuroradiologists (F.E.M. 15 years of experience, and R.R.C. 9 years of experience).

Normalized ADC ratios were computed as the ratio of the ADC values within a lesion to the ADC values within normal white matter. Subsequently, 3D Slicer's Label Statistics function was used to obtain minimum, maximum, mean, standard deviation, skewness, kurtosis, volume, and the percentile values (1st, 5th, 15th, 25th, 75th, 95th, and 99th) of the ADC map VOIs. Qualitative PCNSL characteristics, such as the locations of tumors, the presence of intra-tumor hemorrhage, and the enhancement characteristics on T1-weighted post-gadolinium contrast imaging of tumors, were reviewed by a board-certified neuroradiologist (F.E.M.).

\subsection{Clinical Outcomes}

Two clinical outcomes, overall survival and progression free survival, were analyzed. Complete remission was defined according to the International Primary Central Nervous System Lymphoma Collaboration Group Response Criteria [11,35]. Patients who did not receive treatment were excluded from this analysis. We calculated the OS from the time of diagnosis to death of any cause.

\subsection{Statistical Analysis}

Comparisons of demographic data by HIV status was performed using chi-squared test and fishers exact test when any group contained five or less individuals. Median survival time was generated using Kaplan-Meier methods and $p$-values were calculated using Cox proportional hazards models. Outlier values in ADC mean from white matter images were detected using the Grubb's test and four individuals were excluded from further analyses of imaging data. Normalized ADC ratios were generated by dividing the tumor value against the corresponding white matter value to correct for variations in imaging technologies within the dataset $[10,12,23]$. The association between qualitative imaging characteristics, OS, and PFS was compared using univariable and multivariable Cox proportional hazards models adjusted for age at diagnosis, ECOG score, HIV status, and treatment 
pattern. Treatment pattern was included as a categorical carriable using the following levels: supportive/palliative, whole brain radiation therapy, methotrexate monotherapy, methotrexate-based combination chemotherapy, and whole brain radiation therapy plus methotrexate-based combination chemotherapy. Statistical analyses were performed using R 3.5.0 [36]. Statistical significance was set as $p$-value $<0.05$.

\section{Conclusions}

Our results show promise that ADC histogram values and tumor characteristics may be used by radiologists, after further research and confirmation, to predict the degree of tumor proliferation and patient survival in both immunocompetent patients with PCNSL and PCNSL in PLWH. As new treatment regimens for PCNSL are being trialed, it is important to add to the growing and exciting body of work that investigates the imaging findings of PCNSL, relationships between the imaging and the immunohistochemistry of PCNSL and the correlation between the imaging and patient outcomes.

Supplementary Materials: The following are available online at http://www.mdpi.com/2072-6694/11/10/1506/s1, Table S1: Summary of mean values of ADC histogram parameters, Table S2: Correlations of non-normalized ADC values with $\mathrm{Ki}-67$ in patients with available data.

Author Contributions: Conceptualization: F.M. Data curation: I.C., B.K., D.D. Formal analysis: Q.O., F.M. Investigation: I.C., Q.O., B.K., F.M. Methodology: A.K., R.C., F.M. Project administration: F.M. Resources: N.G., A.K., R.C., F.M. Software: A.K. Supervision: F.M. Validation: Q.O., F.M. Visualization: Q.O. Writing-original draft: I.C., Q.O., B.K., D.D., F.M. Writing-reviewing and editing I.C., Q.O., B.K., D.D., N.G., A.K., R.C., F.M.

Funding: This research received no external funding.

Conflicts of Interest: The authors declare no conflict of interest.

\section{References}

1. Deckert, M.; Montesinos-Rongen, M.; Brunn, A.; Siebert, R. Systems biology of primary CNS lymphoma: from genetic aberrations to modeling in mice. Acta Neuropathol. 2014, 127, 175-188. [CrossRef]

2. Phillips, E.H.; Fox, C.P.; Cwynarski, K. Primary CNS lymphoma. Curr. Hematol. Malig. Rep. 2014, 9, $243-253$. [CrossRef]

3. Bayraktar, S.; Bayraktar, U.D.; Ramos, J.C.; Stefanovic, A.; Lossos, I.S. Primary CNS lymphoma in HIV positive and negative patients: comparison of clinical characteristics, outcome and prognostic factors. J. Neurooncol. 2011, 101, 257-265. [CrossRef] [PubMed]

4. Xie, H.; Ahluwalia, M.S.; Peereboom, D.M. The Cleveland Clinic experience with primary central nervous system lymphoma. Am. J. Clin. Oncol. 2015, 38, 140-146. [CrossRef] [PubMed]

5. Gaudio, F.; Giordano, A.; Perrone, T.; Pastore, D.; Curci, P.; Delia, M.; Napoli, A.; de' Risi, C.; Spina, A.; Ricco, R.; et al. High Ki67 index and bulky disease remain significant adverse prognostic factors in patients with diffuse large B cell lymphoma before and after the introduction of rituximab. Acta Haematol. 2011, 126, 44-51. [CrossRef] [PubMed]

6. Surov, A.; Meyer, H.J.; Wienke, A. Associations between apparent diffusion coefficient (ADC) and KI 67 in different tumors: a meta-analysis. Part 1: ADCmean. Oncotarget 2017, 8, 75434-75444. [CrossRef]

7. Surov, A.; Meyer, H.J.; Wienke, A. Correlation between apparent diffusion coefficient (ADC) and cellularity is different in several tumors: a meta-analysis. Oncotarget 2017, 8, 59492-59499. [CrossRef]

8. Cho, U.; Oh, W.J.; Hong, Y.K.; Lee, Y.S. Prognostic Significance of High Ki-67 Index and Histogenetic Subclassification in Primary Central Nervous System Lymphoma. Appl. Immunohistochem. Mol. Morphol. 2018, 26, 254-262. [CrossRef]

9. Liu, J.; Wang, Y.; Liu, Y.; Liu, Z.; Cui, Q.; Ji, N.; Sun, S.; Wang, B.; Wang, Y.; Sun, X.; et al. Immunohistochemical profile and prognostic significance in primary central nervous system lymphoma: Analysis of 89 cases. Oncol. Lett. 2017, 14, 5505-5512. [CrossRef]

10. Guo, A.C.; Cummings, T.J.; Dash, R.C.; Provenzale, J.M. Lymphomas and high-grade astrocytomas: comparison of water diffusibility and histologic characteristics. Radiology 2002, 224, 177-183. [CrossRef]

11. Han, C.H.; Batchelor, T.T. Primary Central Nervous System Lymphoma. Continuum 2017, 23, $1601-1618$. [CrossRef] [PubMed] 
12. Zhang, Y.; Zhang, Q.; Wang, X.X.; Deng, X.F.; Zhu, Y.Z. Value of pretherapeutic DWI in evaluating prognosis and therapeutic effect in immunocompetent patients with primary central nervous system lymphoma given high-dose methotrexate-based chemotherapy: ADC-based assessment. Clin. Radiol. 2016, 71, 1018-1029. [CrossRef] [PubMed]

13. Wieduwilt, M.J.; Valles, F.; Issa, S.; Behler, C.M.; Hwang, J.; McDermott, M.; Treseler, P.; O’Brien, J.; Shuman, M.A.; Cha, S.; et al. Immunochemotherapy with intensive consolidation for primary CNS lymphoma: a pilot study and prognostic assessment by diffusion-weighted MRI. Clin. Cancer Res. 2012, 18, 1146-1155. [CrossRef] [PubMed]

14. Padhani, A.R.; Liu, G.; Mu-Koh, D.; Chenevert, T.L.; Thoeny, H.C.; Takahara, T.; Dzik-Jurasz, A.; Ross, B.D.; Cauteren, M.V.; Collins, D.; et al. Diffusion-Weighted Magnetic Resonance Imaging as a Cancer Biomarker: Consensus and Recommendations. Neoplasia 2009, 11, 102-125. [CrossRef]

15. Deckert, M.; Brunn, A.; Montesinos-Rongen, M.; Terreni, M.R.; Ponzoni, M. Primary lymphoma of the central nervous system-a diagnostic challenge. Hematol. Oncol. 2014, 32, 57-67. [CrossRef]

16. Koubska, E.; Weichet, J.; Malikova, H. Central nervous system lymphoma: A morphological MRI study. Neuro Endocrinol. Lett. 2016, 37, 318-324.

17. Tang, Y.Z.; Booth, T.C.; Bhogal, P.; Malhotra, A.; Wilhelm, T. Imaging of primary central nervous system lymphoma. Clin. Radiol. 2011, 66, 768-777. [CrossRef]

18. Thurnher, M.M.; Rieger, A.; Kleibl-Popov, C.; Settinek, U.; Henk, C.; Haberler, C.; Schindler, E. Primary central nervous system lymphoma in AIDS: A wider spectrum of CT and MRI findings. Neuroradiology 2001, 43, 29-35. [CrossRef]

19. Le Bihan, D. Apparent Diffusion Coefficient and Beyond: What Diffusion MR Imaging Can Tell Us about Tissue Structure. Radiology 2013, 268, 318-322. [CrossRef]

20. Barajas, R.F., Jr.; Rubenstein, J.L.; Chang, J.S.; Hwang, J.; Cha, S. Diffusion-weighted MR imaging derived apparent diffusion coefficient is predictive of clinical outcome in primary central nervous system lymphoma. AJNR Am. J. Neuroradiol. 2010, 31, 60-66. [CrossRef]

21. Schob, S.; Meyer, J.; Gawlitza, M.; Frydrychowicz, C.; Muller, W.; Preuss, M.; Bure, L.; Quaschling, U.; Hoffmann, K.T.; Surov, A. Diffusion-Weighted MRI Reflects Proliferative Activity in Primary CNS Lymphoma. PLoS One 2016, 11, e0161386. [CrossRef] [PubMed]

22. Schob, S.; Munch, B.; Dieckow, J.; Quaschling, U.; Hoffmann, K.T.; Richter, C.; Garnov, N.; Frydrychowicz, C.; Krause, M.; Meyer, H.J.; et al. Whole Tumor Histogram-profiling of Diffusion-Weighted Magnetic Resonance Images Reflects Tumorbiological Features of Primary Central Nervous System Lymphoma. Transl. Oncol. 2018, 11, 504-510. [CrossRef] [PubMed]

23. Valles, F.E.; Perez-Vallez, C.L.; Regaldo, S.; Barajas, R.F., Jr.; Rubenstein, J.L.; Cha, S. Combined diffusion and perfusion MR imaging as biomarkers of prognosis in immunocompetent patients with primary central nervous system lymphoma. AJNR Am. J. Neuroradiol. 2013, 34, 35-40. [CrossRef] [PubMed]

24. Ahn, S.J.; Shin, H.J.; Chang, J.H.; Lee, S.K. Differentiation between primary cerebral lymphoma and glioblastoma using the apparent diffusion coefficient: comparison of three different ROI methods. PLoS One 2014, 9, e112948. [CrossRef] [PubMed]

25. Fedorov, A.; Beichel, R.; Kalpathy-Cramer, J.; Finet, J.; Fillion-Robin, J.C.; Pujol, S.; Bauer, C.; Jennings, D.; Fennessy, F.; Sonka, M.; et al. 3D Slicer as an image computing platform for the Quantitative Imaging Network. Magn. Reson. Imaging 2012, 30, 1323-1341. [CrossRef]

26. Huang, W.Y.; Wen, J.B.; Wu, G.; Yin, B.; Li, J.J.; Geng, D.Y. Diffusion-Weighted Imaging for Predicting and Monitoring Primary Central Nervous System Lymphoma Treatment Response. AJNR Am. J. Neuroradiol. 2016, 37, 2010-2018. [CrossRef]

27. Whang, J.S.; Kolber, M.; Powell, D.K.; Libfeld, E. Diffusion-weighted signal patterns of intracranial haemorrhage. Clin. Radiol. 2015, 70, 909-916. [CrossRef]

28. Silvera, S.; Oppenheim, C.; Touze, E.; Ducreux, D.; Page, P.; Domigo, V.; Mas, J.L.; Roux, F.X.; Fredy, D.; Meder, J.F. Spontaneous intracerebral hematoma on diffusion-weighted images: influence of T2-shine-through and T2-blackout effects. AJNR Am. J. Neuroradiol. 2005, 26, 236-241.

29. Besusparis, J.; Plancoulaine, B.; Rasmusson, A.; Augulis, R.; Green, A.R.; Ellis, I.O.; Laurinaviciene, A.; Herlin, P.; Laurinavicius, A. Impact of tissue sampling on accuracy of Ki67 immunohistochemistry evaluation in breast cancer. Diagn. Pathol. 2016, 11, 82. [CrossRef] 
30. Go, J.L.; Lee, S.C.; Kim, P.E. Imaging of primary central nervous system lymphoma. Neurosurg. Focus 2006, 21, E4. [CrossRef]

31. Haldorsen, I.S.; Espeland, A.; Larsson, E.M. Central nervous system lymphoma: Characteristic findings on traditional and advanced imaging. AJNR Am. J. Neuroradiol. 2011, 32, 984-992. [CrossRef] [PubMed]

32. Zacharia, T.T.; Law, M.; Naidich, T.P.; Leeds, N.E. Central nervous system lymphoma characterization by diffusion-weighted imaging and MR spectroscopy. J. Neuroimaging 2008, 18, 411-417. [CrossRef] [PubMed]

33. Dandachi, D.; Ostrom, Q.T.; Chong, I.; Serpa, J.A.; Giordano, T.P.; Kruchko, C.; Barnholtz-Sloan, J.S.; Fowler, N.; Colen, R.R.; Moron, F.E. Primary central nervous system lymphoma in patients with and without HIV infection: a multicenter study and comparison with U.S national data. Cancer Causes Control 2019, 30, 477-488. [CrossRef] [PubMed]

34. Zhu, J.; Zhang, J.; Gao, J.Y.; Li, J.N.; Yang, D.W.; Chen, M.; Zhou, C.; Yang, Z.H. Apparent diffusion coefficient normalization of normal liver: Will it improve the reproducibility of diffusion-weighted imaging at different MR scanners as a new biomarker? Medicine (Baltimore) 2017, 96, e5910. [CrossRef] [PubMed]

35. Abrey, L.E.; Batchelor, T.T.; Ferreri, A.J.; Gospodarowicz, M.; Pulczynski, E.J.; Zucca, E.; Smith, J.R.; Korfel, A.; Soussain, C.; DeAngelis, L.M.; et al. Report of an international workshop to standardize baseline evaluation and response criteria for primary CNS lymphoma. J. Clin. Oncol. 2005, 23, 5034-5043. [CrossRef]

36. R Core Team. R: A Language and Environment for Statistical Computing. Available online: http: //www.R-project.org/ (accessed on 22 April 2018).

(C) 2019 by the authors. Licensee MDPI, Basel, Switzerland. This article is an open access article distributed under the terms and conditions of the Creative Commons Attribution (CC BY) license (http://creativecommons.org/licenses/by/4.0/). 